PAPER

\section{High sensitivity LPG Mach-Zehnder sensor for real-time fuel conformity analysis}

To cite this article: Jonas H Osório et al 2013 Meas. Sci. Technol. 24015102

View the article online for updates and enhancements.

\section{Related content}

Application of a long-period fibre gratingbased transducer in the fuel industry G R C Possetti, L C Côcco, C I Yamamoto et al.

Refractometric sensor based on all-fiber coaxial Michelson and Mach-Zehnder interferometers for ethanol detection in

L Mosquera, Jonas H Osório, Juliano G Hayashi et al.

Detection of adulteration in virgin olive oil using a fiber optic long period arating based sensor

T M Libish, M C Bobby, J Linesh et al.

\section{Recent citations}

- Highly sensitive refractive index sensor
$\frac{\text { based on degeneracy in specialty optical }}{\text { fibers: a new approach }}$
Arpan Roy et al
- Physical measurement with in-line fiber
$\frac{\text { Mach-Zehnder interferometer using }}{\text { differential phase white light interferometry }}$
Seyed Hashem Aref
- Integration of bow-tie plasmonic nano-
$\frac{\text { antennas on tapered fibers }}{\text { Abdul Khaleque et al }}$




\title{
High sensitivity LPG Mach-Zehnder sensor for real-time fuel conformity analysis
}

\author{
Jonas H Osório $^{1}$, L Mosquera ${ }^{1}$, Carlos J Gouveia ${ }^{2}$, Claudecir R Biazoli ${ }^{1}$, \\ Juliano G Hayashi ${ }^{1}$, Pedro A S Jorge ${ }^{2}$ and Cristiano M B Cordeiro ${ }^{1}$ \\ ${ }^{1}$ Instituto de Física Gleb Wataghin, UNICAMP, Campinas, SP, Brazil \\ 2 INESC-Porto, Rua do Campo Alegre, 687, 4169-007 Porto, Portugal \\ E-mail: jhosorio@ifi.unicamp.br and cmbc@ifi.unicamp.br
}

Received 30 July 2012, in final form 15 October 2012

Published 10 December 2012

Online at stacks.iop.org/MST/24/015102

\begin{abstract}
A high sensitivity refractive index sensor based on the combination of mechanically induced long period gratings (LPG) and fiber tapers was developed for real-time fuel quality analysis. The sensor was built in a Mach-Zehnder configuration by employing a pair of in-series gratings. In order to enhance sensor sensitivity, the region between both LPGs was tapered down from 125 to $10 \mu \mathrm{m}$. The system was tested by measuring water concentration in ethanol and ethanol concentration in commercial gasoline. The tapered sensor has shown an average sensitivity of $930 \mathrm{~nm} / \mathrm{RIU}, 18$ times higher than the non-tapered version. The resolution limit of the system using spectral interrogation was estimated to be $0.06 \%$ of ethanol dissolved in gasoline. For the purpose of real-time monitoring, an interrogation system based on white light interferometry (WLI) and virtual instrumentation was employed to evaluate ethanol evaporation in water, avoiding the use of spectral analysis. The WLI system, using phase tracking techniques, enabled us to record the evolution of the ethanol concentration in water with a resolution of $0.005 \%(\mathrm{v} / \mathrm{v})$.
\end{abstract}

Keywords: fiber optics, sensors, long period grating, fiber taper, fuel

(Some figures may appear in colour only in the online journal)

\section{Introduction}

Gasoline is a complex mixture of liquid hydrocarbons derived from petroleum, volatile and flammable, with special physical and chemical properties that allow its applicability in the field of fuels, especially as fuel for internal-combustion engines. The exact composition of gasoline varies with the characteristics of the raw material, its production process and on the specifications imposed by regulatory agencies. In Brazil, for instance, according to the government laws the distributed and commercialized gasoline contains anhydrous ethanol, with a volume percentage between $20 \%$ and $25 \%$. Ethanol-gasoline blend has some advantages over the pure gasoline blend. For instance, the inclusion of ethanol improves the thermodynamic efficiency of the product providing better performance for combustion engines. Furthermore, the combustion of ethanol- gasoline blend provides significant reduction in the emission of air pollutants. Finally, a relevant fact is that the ethanol stems from fermented biomass, a renewable natural resource $[1,2]$.

However, one of the biggest problems with this practice is that the sector has to deal with fuel adulteration. A common malpractice is to increase the solvent and/or ethanol concentration in proportions higher than those stated by the legislation. Fuel quality monitoring requires the application of a rigorous sampling technique in conjunction with several and expensive analyses of fuel physical and chemical properties. On the other hand, the use of alternative and renewable energy sources has drawn great attention in recent years [1,2].

In this context, ethanol is one of the most common alternatives. Whilst ethanol can be considered an environmentally friendly compound, it is a versatile solvent 
miscible in all proportions with water. Consequently, operating an engine with an ethanol fuel with a high water percentage or with a gasoline blend with too high hydrated alcohol content can cause engine damage and poor performance, with possible consequences to the environment. Therefore, a careful monitoring of water content in ethanol fuel is necessary to avoid these problems. Within this context, the development of smart monitoring systems able to assess the fuel conformity and to supply real-time and reliable results assumes great importance $[1,2]$.

Fiber optic sensors are an interesting solution in this context due to their high sensitivity, small size, electromagnetic immunity, electrical passivity, non-chemical reactivity and capability for in situ, real-time and remote sensing. Long period gratings (LPG), in particular, can be used as refractive index sensors, being useful for such purposes.

LPGs consist of a periodic perturbation in optical fiber refractive index which is able to, at specific wavelengths, partially couple light that propagates in the fundamental core mode to co-propagating cladding modes [3]. Such cladding modes can have interesting chromatic dispersion characteristics [4, 5] or, more generally, be used for sensing applications. Grating transmission spectrum, which is characterized by attenuation bands due to the high loss experienced by the cladding modes, can be used to sense parameters such as temperature, LPG surrounding medium refractive index and fiber bending curvatures [6].

LPGs can be fabricated by employing different techniques which can provide the necessary changes in the fiber refractive index. Photoinduced LPGs can be produced by exposing the fiber to UV laser irradiation using an amplitude mask or a point-by-point technique. LPG fabrication techniques based on the use of $\mathrm{CO}_{2}$ lasers, on fiber exposure to arc discharges or fiber subjection to mechanical stress have also been developed [7-11].

Mechanically induced long-period fiber gratings (MLPGs) have been attractive due to their simplicity, flexibility and low cost. Further, MLPGs, which can be recorded on single mode fibers [9], holey fibers [10] or even on tapered fibers [11], can have their attenuation loss controlled in real time, what makes them very promising for tunable spectral filtering, gain equalization and other applications.

Among the possible applications referenced above are refractive index sensors. As the grating makes the light propagate through fiber cladding, it becomes sensitive to external environment refractive index-since the light evanescent field will permeate the region around the fiber. Therefore, it is possible to apply the LPG-based devices to probe the external environment-making them a suitable platform for the development of sensors for fuel conformity analysis.

In order to improve the resolution of these sensors, it is possible to set the LPGs to act as a beam splitter or combiner in an all-fiber interferometer configuration. Literature reports configurations based on the use of two LPGs [12-14], in which the first LPG couples part of the core mode energy to cladding modes and the second makes the light which propagates through the cladding re-couple in the core. Also, in a reflection measurement configuration, just one grating can be employed-in this case, the fiber end tip must be metalized [15-18].

Likewise, sensor sensitivity can be improved by tapering down the fiber region between two LPGs [13, 14]. Fiber tapering causes a greater portion of cladding mode to extend beyond the fiber interface as evanescent field. This lowers the propagating mode effective refractive index making it more sensitive to the external medium refractive index changes.

Fiber optic sensors for fuel conformity analysis reported so far are mostly based on simple LPG configurations [19]. Just very recently, an all-fiber LPG interferometer device was studied for the assessment of fuel refractive index [1]. In particular, a Michelson configuration using a simple LPG and a mirrored tip was used.

In this work, a more versatile solution is proposed using MLPG based interferometers. Different configurations are studied including the case in which a fiber with reduced diameter was employed in order to increase system sensitivity. A practical application based on detecting percentages of water dissolved in ethanol and of ethanol dissolved in commercial gasoline will be shown. Also, to demonstrate the system ability for real-time high sensitivity monitoring, ethanol evaporation was evaluated while using a white light interferometry (WLI) interrogation system with virtual instrumentation in a differential configuration.

\section{Mechanically induced long period gratings}

Gratings were mechanically induced on commercial standard single mode optical fiber. Figure 1( $a$ ) shows the experimental setup used to produce the MLPGs where a V-grooved plastic board was used to press the fiber. MLPG transmission spectra were measured by launching broadband light (supercontinuum generated in a photonic crystal fiber) into the fiber and by connecting the fiber end tip to an optical spectrum analyzer (OSA Ando AQ-6315).

Figure $1(b)$ shows the transmission spectrum of a MLPG $20 \mathrm{~mm}$ long and $500 \mu \mathrm{m}$ pitch-firstly the fiber spectrum without the grating is measured; afterwards, the grating spectrum is measured and then the two curves are subtracted. The observed dips at 1473 and $1549 \mathrm{~nm}$ correspond to cladding mode resonances. Phase matching conditions between the fundamental core mode and specific forward-propagating cladding modes are achieved at wavelengths $\lambda_{n}$ which satisfy the following equation [3]:

$$
\lambda_{n}=\left(n_{\text {co }}^{01}-n_{\text {clad }}^{0 n}\right) \Lambda
$$

where $n_{\mathrm{co}}^{01}$ is the effective core mode refractive index, $n_{\mathrm{clad}}^{0 n}$ is the effective refractive index of the $n$th order cladding mode and $\Lambda$ is the LPG period (distance between the points that pressurize the fiber). One can note that the coupled cladding modes have zero azimuthal order $(l=0)$. This happens because the coupling must occur between modes that result in a non-zero overlap integral. Thus, the coupled cladding modes must have light in the core region of the fiber-which is a characteristic of $l=0$ modes.

Figure 1(b) also presents MLPG transmission spectrum dependence on the applied pressure on the fiber. One can 


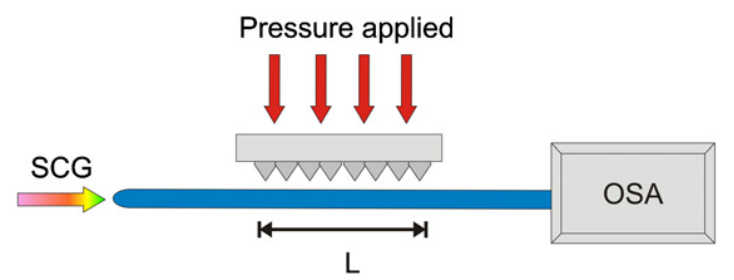

(a)

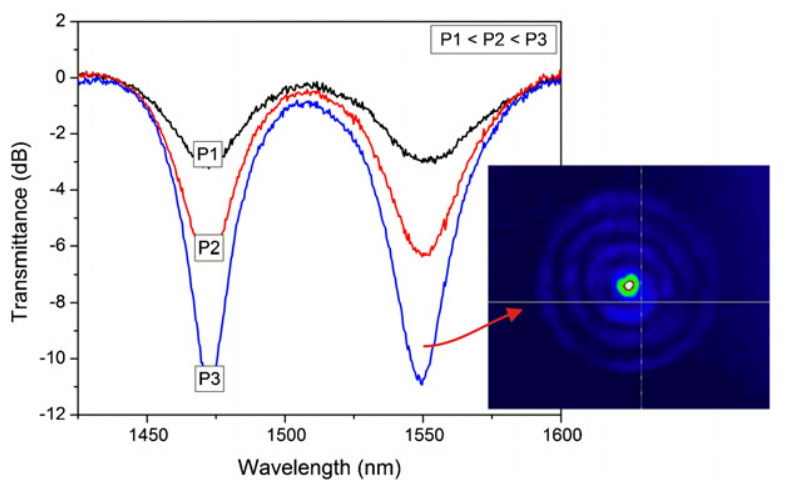

(b)

Figure 1. (a) Experimental setup for MLPG fabrication. SCG: supercontinuum generation in photonic crystal fibers, $L$ : grating length. (b) MPLG transmission spectrum as a function of the applied pressure $(L=20 \mathrm{~mm} ; \Lambda=500 \mu \mathrm{m})$. Inset shows the out-coupled cladding mode near-field pattern at $\lambda=1549 \mathrm{~nm}$.

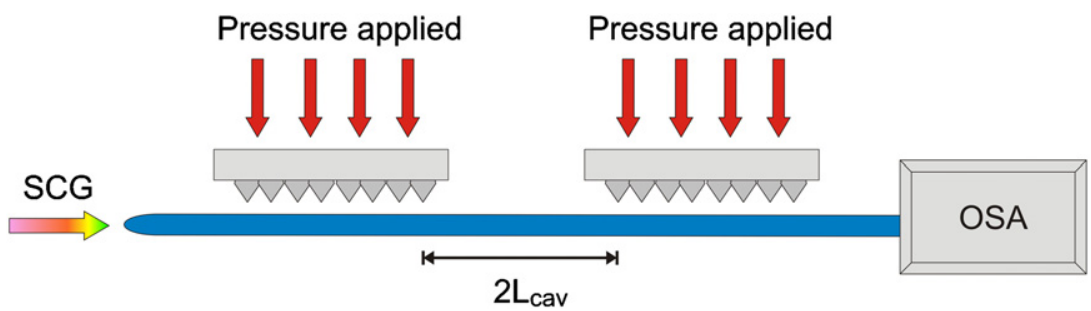

Figure 2. Schematic diagram for the MLPG Mach-Zehnder interferometer.

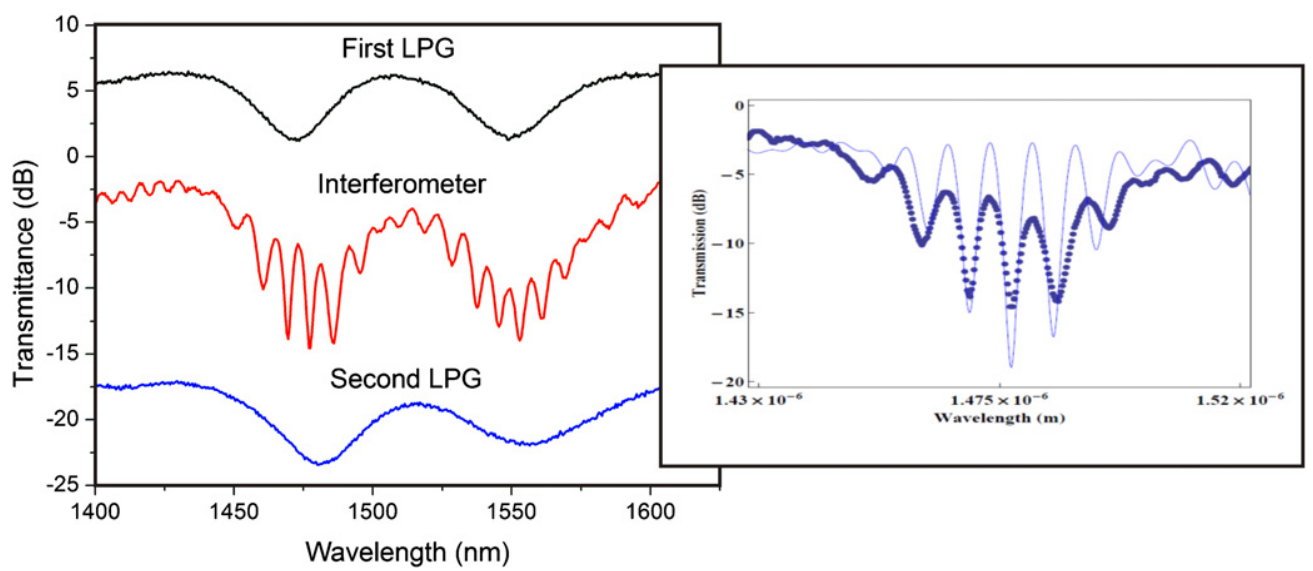

Figure 3. MLPGs individual and associated spectra are shown. Inset shows a simulation (continuous line) for Mach-Zehnder interferometer transmission spectrum using the following parameters: $\Lambda=500 \mu \mathrm{m} ; \Delta n_{1}=2.955 \times 10^{-3} ; \Delta n_{2}=3.080 \times 10^{-3} ; L_{1}=19 \mathrm{~mm}$; $L_{2}=19 \mathrm{~mm}$ and $2 L_{\mathrm{cav}}=40 \mathrm{~mm}$. Experimental data reproduced for better comparison.

observe that when pressure is increasingly applied on the fiber, transmission loss is increased to approximately $11 \mathrm{~dB}$. The attenuation band depth depends on a squared sine which has the coupling coefficient as its argument. Thus, if the applied pressure is high enough, resonance depth increases to a maximum value and then decreases-meaning that the mode is being re-coupled to the core.

In order to identify the coupled cladding modes, mode imaging was performed. A tunable laser was used to couple light in the core mode and, by tuning the laser wavelength, a cladding mode was selectively excited and the transmitted light was projected on an infrared camera (Micron Viewer) using a near-field technique. The inset in figure $1(b)$ shows the near-field output pattern obtained by exciting $\mathrm{LP}_{06}$ mode.

\section{Interferometer characterization}

Firstly, an all-fiber Mach-Zehnder interferometer (MZI) based on a pair of in-series MLPGs was implemented (figure 2). In this setup, a fraction of the power in the core mode is transferred to the cladding modes by the first MLPG. Therefore, in the region between the two MLPGs, light propagates through core and cladding simultaneously. In sequence, the second MLPG induces coupling between the cladding to the core mode and a spectral fringe pattern appears in the transmission spectrum.

The interferometer was produced by employing a pair of MLPGs with period $\Lambda=500 \mu \mathrm{m}$ and length $L=20 \mathrm{~mm}$ which were $40 \mathrm{~mm}$ apart. Figure 3 shows the LPGs transmission 


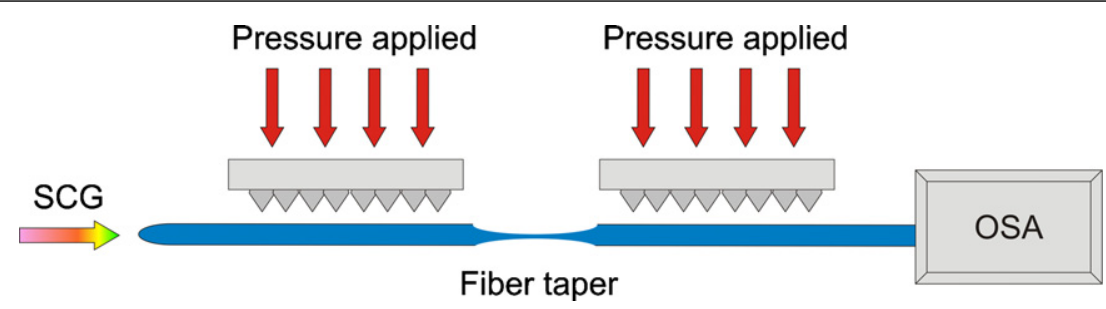

Figure 4. Schematic diagram for the MLPG Mach-Zehnder interferometric sensor with a fiber taper between the gratings.

spectrum when they act alone and when they act togetherfigures are vertically shifted for better visualization.

The inset in figure 3 shows a simulation for interferometer transmission spectrum using $\Lambda=500 \mu \mathrm{m} ; \Delta n_{1}=2.955 \times$ $10^{-3} ; \Delta n_{2}=3.080 \times 10^{-3} ; L_{1}=19 \mathrm{~mm} ; L_{2}=19 \mathrm{~mm}$ and $2 L_{\mathrm{CAV}}=40 \mathrm{~mm}$. The index differences $\Delta n_{1}$ and $\Delta n_{2}$ were simulated with values close to those expected from equation (1)—using $\lambda_{n}=1473 \mathrm{~nm}$ and $\Lambda=500 \mu \mathrm{m}$-in order to obtain the best agreement with experimental data.

In this MLPG configuration, the separation between them $\left(2 L_{\text {cav }}\right)$ determines the number of interferometric fringes in the transmission spectrum, that is, the increase of gratings separation causes the fringes to be spectrally closer. Experimental data showed that for $L_{\mathrm{cav}}=35 \mathrm{~mm}$, fringes were typically $17 \mathrm{~nm}$ apart and for $L_{\text {cav }}=108 \mathrm{~mm}, 7 \mathrm{~nm}$ apart.

Considering this, the choice of cavity length must consider measurement dynamic range (i.e. the refractive index interval to be evaluated) besides measurement resolution. This consideration is important because, as an increase in cavity length causes the fringes to be spectrally closer, ambiguity may occur when interpreting sensor response. This happens because there is a compromise between resolution and dynamic range, the use of long cavities being recommended only when measuring short refractive index intervals with high sensitivity. It should be noted that tuning the cavity length is straightforward when dealing with mechanically induced gratings.

The setup presented has the great advantage of being extremely simple and inexpensive because it employs standard telecom optical fibers and machinable corrugated boards. It also presents the possibility of tuning its spectral response by adjusting the applied pressure, grating and cavity lengths. However, the sensitivity of such devices to external refractive index, which is highly dependent on the mode order and on the external refractive index, is usually low, typically in the order of $30 \mathrm{~nm} / \mathrm{RIU}$ for refractive index changes around 1.33 [13] and $230 \mathrm{~nm} / \mathrm{RIU}$ for refractive index changes around 1.42 [19].

To enhance such sensitivity, the fiber region between the gratings can be tapered down as shown in figure 4. In this case, as the fiber taper causes a greater fraction of the cladding mode field to extend beyond the fiber interface (i.e. in the external medium), an expressive increase in sensor sensitivity can be observed. In [13], a 38.75 microns thick taper was included between two laser induced LPGs with the expected sensitivity enhancement. A sensitivity of approximately $172 \mathrm{~nm} / \mathrm{RIU}$ was achieved for refractive index change between 1.3333 and 1.3624 .
Here, we combined mechanically induced all-fiber interferometers based on LPGs and fiber tapers. Also, differently from [13], a thinner taper (10 $\mu \mathrm{m}$ diameter) was used-which allowed us to obtain a sensitivity about four times higher $(695 \mathrm{~nm} / \mathrm{RIU})$ for refractive index change around 1.333 - as well as a WLI setup enabling real-time phase tracking ability with higher resolution. Applications and interrogation schema will be discussed below.

\section{Application: sensors for fuel conformity analysis}

As optical fiber devices present passive operation, they can be useful for determining fuel quality (detecting adulteration, for example). Thus, in this investigation, one has performed sensing experiments concerning water concentration in ethanol and ethanol concentration in commercial gasoline.

Figure 5(a) shows water-ethanol sensor response. Gratings used had $600 \mu \mathrm{m}$ pitch, were $20 \mathrm{~mm}$ long and were $175 \mathrm{~mm}$ distant from each other. Sensor operation is evidenced by the spectral shift of interferometric fringes when the tapered region (15 $\mathrm{mm}$ long and $10 \mu \mathrm{m}$ thick) was immersed in water-ethanol solutions with different concentrations. One can observe that as mixtures refractive index increases, fringes fall at lower wavelengths. From the obtained data, one can plot the fringe wavelength as a function of mixture refractive index (figure $5(b)$ ). A sensitivity of $695 \mathrm{~nm} / \mathrm{RIU}$ can then be calculated. This value is about four times higher in relation to the data reported in [13].

Also, ethanol concentration in commercial gasoline was evaluated. Figure $6(a)$ shows the interferometer response when it acts as an ethanol-gasoline sensor. Gratings with a $600 \mu \mathrm{m}$ pitch and $20 \mathrm{~mm}$ long were used. The distance between the gratings was $410 \mathrm{~mm}$ in order to obtain a large number of interferometric fringes and, consequently, a reduced FWHM $(\Delta \lambda=1 \mathrm{~nm})$. This procedure made it easier to observe fringe shift when the concentration of ethanol in gasoline was changed. Figure $6(b)$ shows the fringes spectral shift as a function of the solution refractive index. This procedure again demonstrated the versatility of the system which enables straightforward adjustment of the sensor characteristics by simple mechanical adjustment.

Again, as mixtures refractive index increased, fringes fell at lower wavelengths. For instance, the fringe centered at $1511.46 \mathrm{~nm}$ shifted to $1509.38 \mathrm{~nm}$ as the refractive index changed from 1.422 to 1.424 .

The refractive index of mixtures $(n)$ of two liquids with refractive indices $n_{1}$ and $n_{2}$ were estimated by equation (2). 


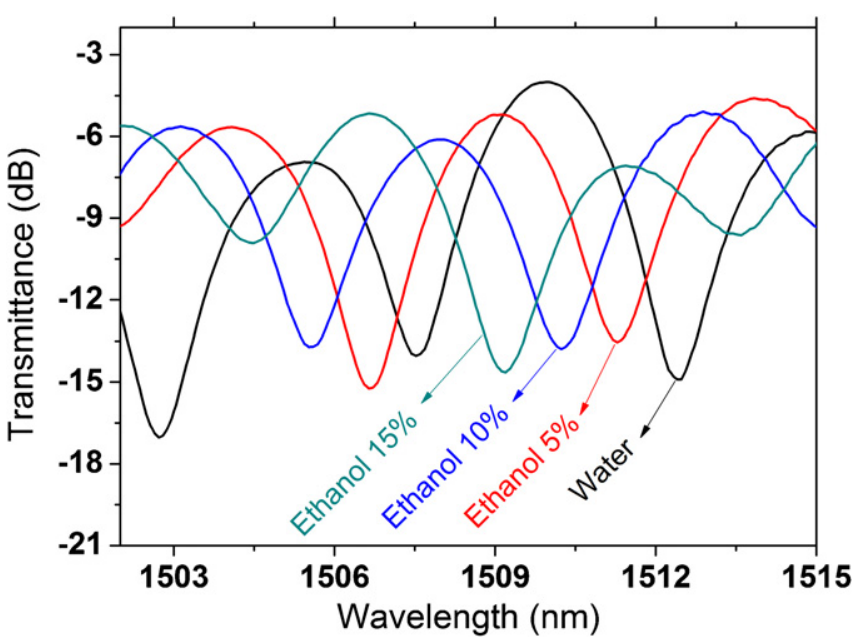

(a)

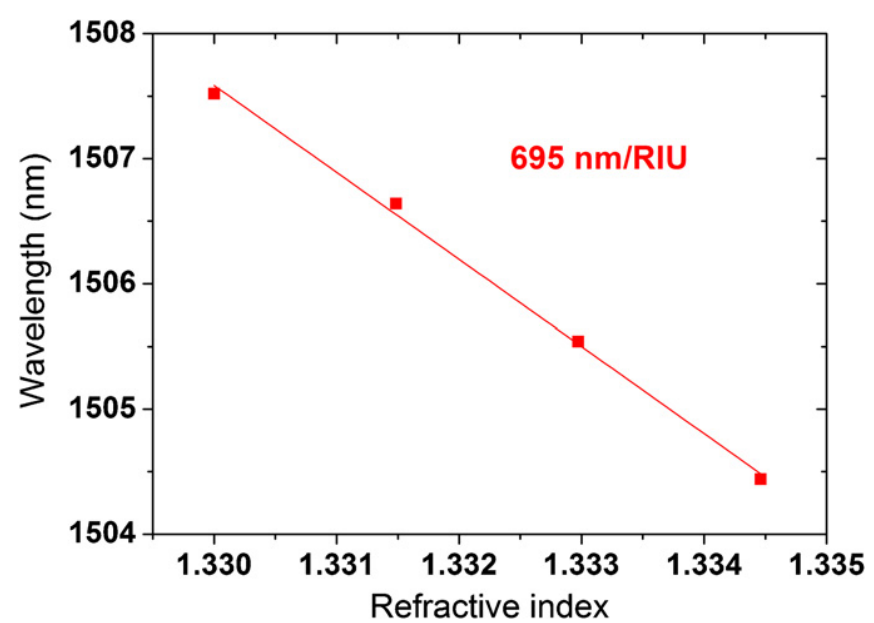

(b)

Figure 5. (a) Water-ethanol sensor response. The configuration of figure 4 was used. Gratings had $600 \mu \mathrm{m}$ pitch and were $20 \mathrm{~mm}$ long. Gratings were $175 \mathrm{~mm}$ apart. (b) Fringe wavelength as a function of the surrounding refractive index. Sensitivity of $695 \mathrm{~nm} / \mathrm{RIU}$ was obtained.

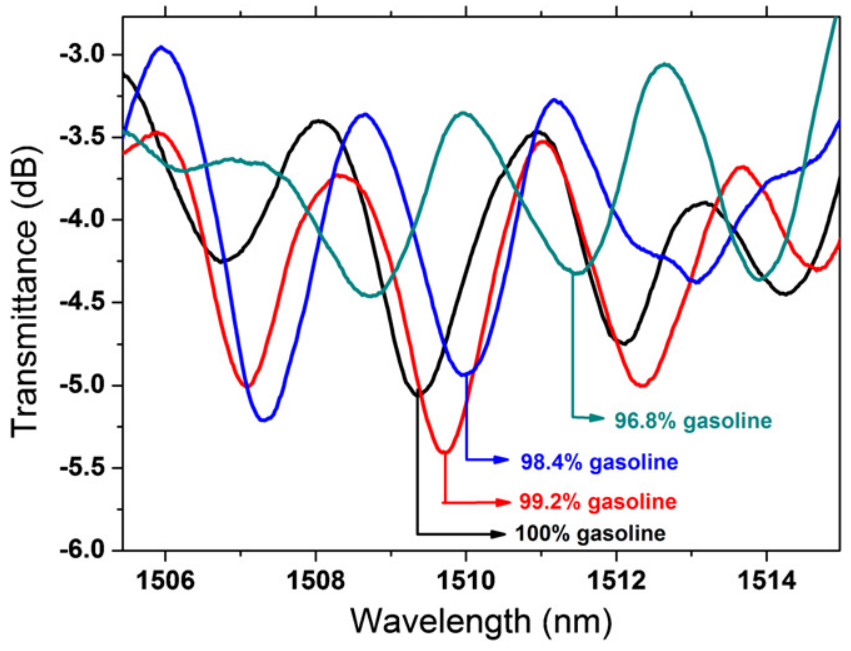

(a)

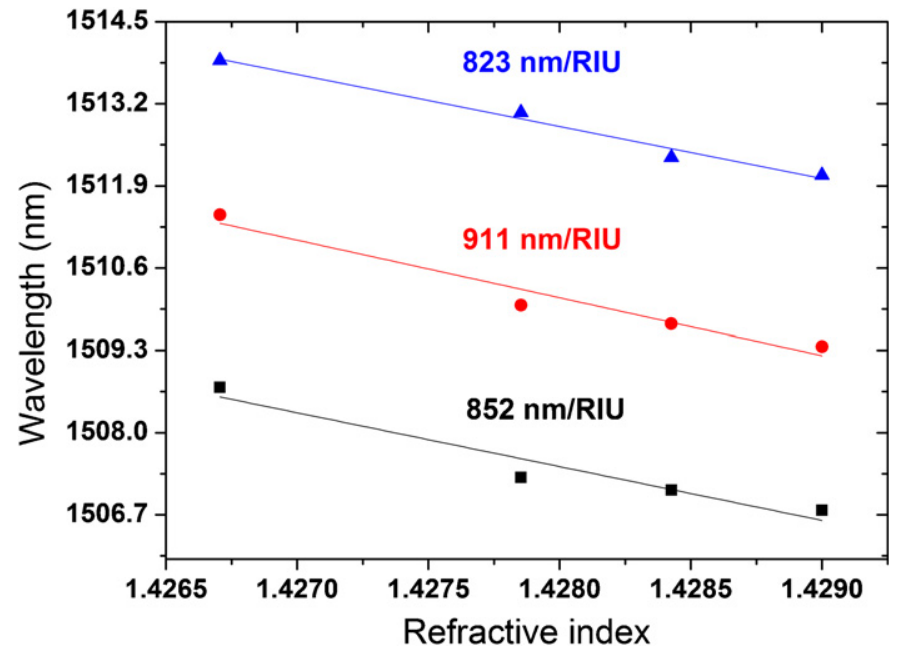

(b)

Figure 6. (a) Spectral shift of interferometric fringes. MLPGs: $\Lambda=600 \mu \mathrm{m} ; L=20 \mathrm{~mm} ; 2 L_{\mathrm{cav}}=410 \mathrm{~mm}$. $(b)$ Fringe wavelength as a function of the refractive index associated with the percentage of ethanol dissolved in commercial gasoline.

$\phi$ in equation (3) means the volume concentration of liquid with refractive index $n_{1}[20]$ :

$$
n=\sqrt{\frac{2 g+1}{1-g}},
$$

where

$$
g=\left(\frac{n_{1}^{2}-1}{n_{1}^{2}+2}\right) \phi+\left(\frac{n_{2}^{2}-1}{n_{2}^{2}+2}\right)(1-\phi) .
$$

Refractive indices used were 1.333 for water, 1.359 for ethanol [21] and 1.424 for Brazilian commercial gasoline [19] — all of them at $589 \mathrm{~nm}$.

Considering all the fringes presented in figure $6(a)$, the sensor average sensitivity is $930 \mathrm{~nm} / \mathrm{RIU}$. In terms of ethanol concentration, the sensitivity can be calculated as $0.62 \mathrm{~nm} / \%$ of ethanol dissolved in gasoline - a sensitivity 18 times higher when compared to $0.035 \mathrm{~nm} / \%$ ethanol obtained without the tapered region (using the configuration referenced in figure 2).
Besides, this sensitivity is fourfold higher when compared to $0.15 \mathrm{~nm} / \%$ ethanol reported in [19].

Also, the achieved sensitivity is $50 \%$ higher when compared to the sensor reported in [22], where the authors tested a refractive index sensor based on Bragg gratings inscribed in microfibers.

Using the FWHM cited above $(\Delta \lambda=1 \mathrm{~nm})$, one can estimate a resolution limit for the developed system considering that one can resolve two fringes if they are at least a wavelength difference of $\Delta \lambda / 25=0.04 \mathrm{~nm}$ apart. This means that the estimated resolution limit of our system would be $0.06 \%$ of ethanol.

Moreover, if an optical spectrum analyzer of spectral resolution of $10 \mathrm{pm}$ is used, in terms of refractive index, a resolution of $1.08 \times 10^{-5}$ can be achieved-a value which has the same order of magnitude as the resolution reported in [23], where the authors studied a refractive index sensor based on Fresnel reflection. 


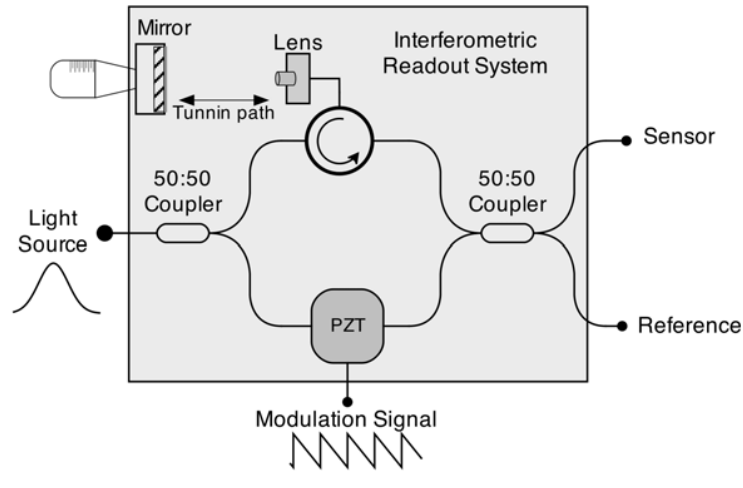

(a)

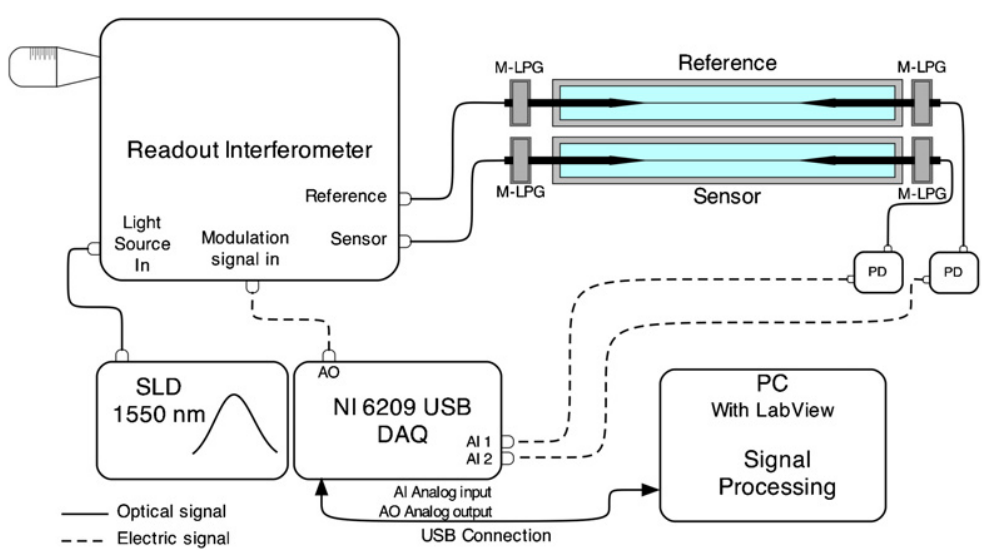

(b)

Figure 7. (a) Readout interferometer interrogation device. (b) Setup for differential measurement of refractive index using readout interferometer and mechanically induced LPG MZI.

We should analyze, however, that this sensor can only detect adulteration if the tampering agents have different refractive index than gasoline. Still, as the main tampering agents-ethanol and kerosene-have considerably different refractive index than gasoline-1.359 [21] and 1.436 [24], respectively - we evaluate that the proposed sensor can indeed be useful.

\section{Real-time concentration measurement with WLI interrogation system}

In an operational context, the use of an optical spectrum analyzer is not practical. In order to avoid spectral acquisition, a WLI technique was used for sensor interrogation in real time. This method provided an interrogation system which allowed tracking the fringes wavelength shift in the form of the phase shift of an electronic carrier signal.

Figure 7(a) shows the setup of the WLI system based on a standard fiber-optic MZI. In one of the arms of the MZI, a piezoelectric phase modulator (PZ1 from Optiphase) was inserted for carrier generation by periodic phase modulation. On the other arm, through a circulator, an air path was implemented using a GRIN lens and a mirror mounted in a translation stage. This arrangement allowed to adjust the interferometer path imbalance and also to modulate its phase as desired. A sawtooth modulation with its amplitude adjusted to obtain a $2 \pi$ phase excursion was used yielding a sinusoidal carrier generation at the interferometer output [25].

For the purpose of evaluating the developed sensor response for refractive indices around 1.33 , one evaluated the ethanol evaporation as a function of time. Figure $7(b)$ shows the experimental setup employed for differential measurement of refractive index. The readout interferometer was illuminated by a SLED source (super light emitting diode, $\Delta \lambda \approx 120 \mathrm{~nm}$ and $\left.L_{\text {coherence }} \approx 10 \mu \mathrm{m}\right)$. A sawtooth modulation signal $(1 \mathrm{kHz})$ with amplitude $4 \mathrm{~V}$ generated by a signal acquisition board (DAQ NI 6259 USB) was applied.

The two output ports of the readout interferometer were used to interrogate two identical MLPG interferometric sensors. For this purpose, the reading interferometer path imbalance was tuned to match the path imbalance of the sensors. In this situation, the phase of the output carrier is proportional to the fringe wavelength shift of each interferometer.

Using one of the interferometers as reference and the other as sensor, we obtained a situation in which environmental perturbations affect the phase of both signals and, by phase subtraction, the common information is canceled out. Therefore, the phase difference between the two signals depends only on the modulation inferred by the parameter of interest allowing a very stable and accurate phase measurement retrieved using phase comparison software $[25,26]$.

The response to the surrounding RI was studied by exposing the sensor to different solutions of distilled water mixed with different concentrations of ethanol to provide the RI standards in a range between 1.338 and 1.356. The calibration curve obtained is represented in figure $8(a)$. Results show, as expected, an increment in the phase difference with the increase of the surrounding refractive index. From the calibration curve, a sensitivity of $23600 \mathrm{deg} /$ RIU was estimated.

The detection limit can be estimated by $2 \sigma$, where $\sigma$ is the highest standard deviation considering all calibration points, divided by the sensitivity. Thus, an average resolution of $1.5 \times 10^{-5}$ was calculated - which is very close to the one obtained in the spectral measurement: $1.4 \times 10^{-5}$ (estimated assuming a resolution of $10 \mathrm{pm}$ and a sensitivity of $685 \mathrm{~nm} / \mathrm{RIU}$ obtained in the previous water ethanol/experiment).

However, it should be mentioned that very seldom is the resolution with which the fringe shift can be tracked given by the spectral instrument maximum resolution. Signal-to-noise ratio and fringe period often result in degraded resolution. In addition, in this particular case the interferometric system, which had a phase stability of $2 \sigma=0.2 \mathrm{deg}$, was not fully optimized. Improving thermal and acoustical insulation, introducing polarization control, etc can easily result in improving this figure by at least an order of magnitude, an improvement that would directly impact the measurement resolution. 


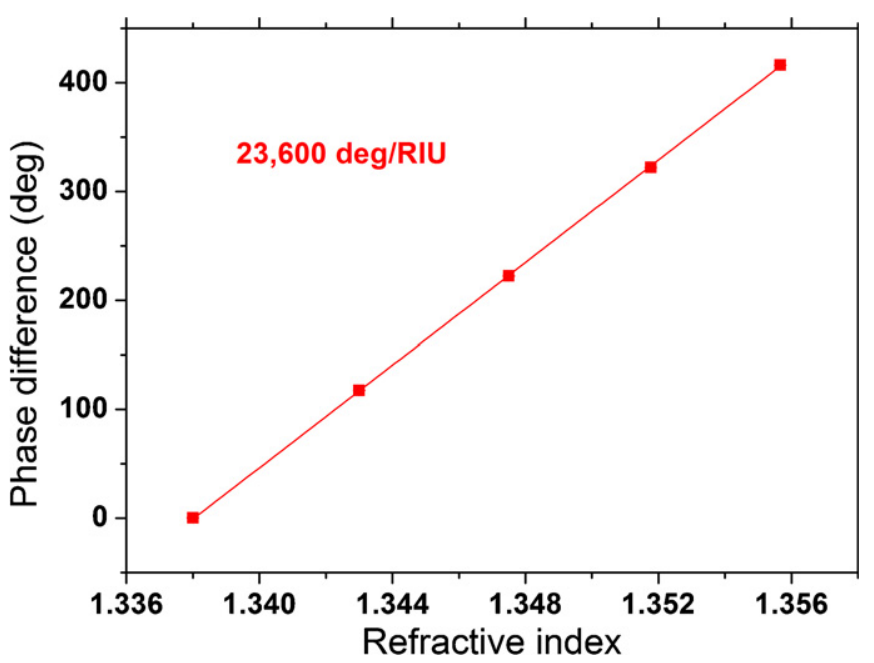

(a)

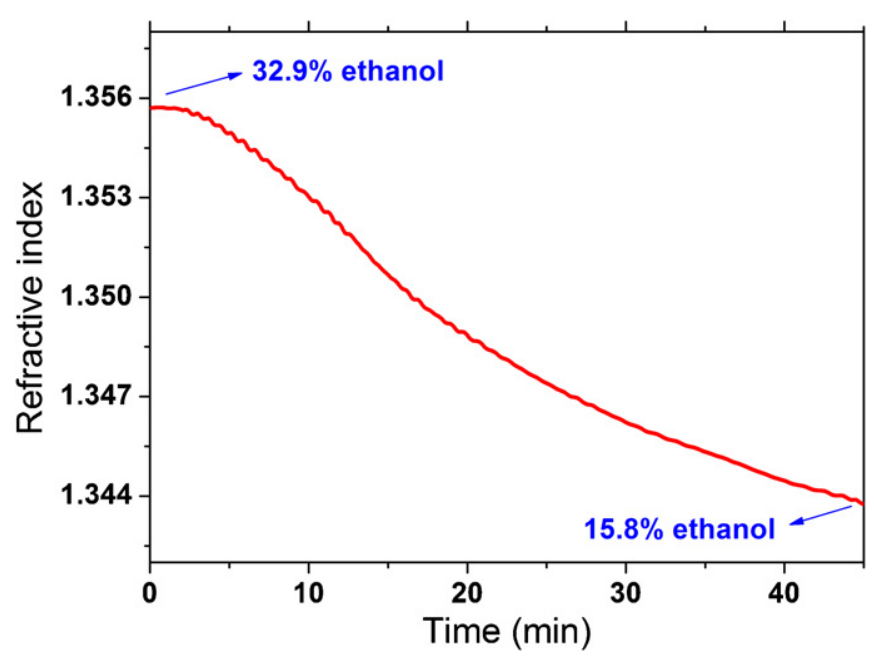

(b)

Figure 8. (a) Calibration curve for refractive index variations. (b) Ethanol evaporation measurement.

Figure $8(b)$ shows the real-time system differential response in a measurement in which ethanol evaporation was evaluated. To do this, a water-ethanol mixture was used to immerse the tapered region of the fiber and a measurement of the phase difference as a function of time was performed. Mixture refractive index was directly obtained by the phase difference (using the calibration curve).

After obtaining the mixture refractive index, equations (2) and (3) can be used to obtain the corresponding ethanol concentration in water. Thus, one could observe that, after $45 \mathrm{~min}$, the concentration of ethanol in the chamber decreased from $32.9 \%$ to $15.8 \%$. From the results obtained, it was estimated that the ethanol concentration could be tracked with a resolution as high as $0.005 \% \mathrm{v} / \mathrm{v}$, demonstrating the ability for real-time assessment of fuel quality with very high resolution.

\section{Conclusion}

In this paper, the development of a high sensitivity sensor using mechanically induced long period gratings and fiber tapers was reported for real time fuel conformity analysis. The mechanical nature of the LPG and the tapering enabled to rapidly adjust the sensor characteristics (fringe period, sensitivity) in a straightforward fashion.

In order to demonstrate sensor versatility and applicability to the fuel industry, measurements were performed in waterethanol and ethanol-gasoline mixtures. Both sensors presented high sensitivity: $695 \mathrm{~nm} / \mathrm{RIU}$ for the water-ethanol sensor and $930 \mathrm{~nm} / \mathrm{RIU}$ for the ethanol-commercial gasoline sensor.

To avoid the use of expensive spectral interrogation devices, the sensors were interrogated using a low-cost white light interferometric interrogation system with virtual instrumentation. Using MLPG allowed us to easily replicate two identical interferometers with exactly matching optical path imbalances. Thus, differential arrangement could be tested using a reference interferometer, immersed in distilled water, and a sensor interferometer, immersed in water-ethanol mixtures. Their relative phase was shown to be practically immune to most environmental perturbations and proportional only to the water/ethanol ratio, enabling real time, long-term measurements with very high accuracy and stability.

The platform demonstrated has a strong potential for other environmental monitoring applications involving label free sensor techniques.

\section{Acknowledgments}

This research was supported by FAPESP, CNPq and CAPES (bi-national collaboration project, CAPES-FCT Brazil/Portugal coordinated by authors CMBC and PJ). Also, the authors would like to thank José Aparecido dos Santos for technical support.

\section{References}

[1] Kawano M S, Heidemann B R, Cardoso T K M, Possetti G R C, Kamikawachi R C, Muller M and Fabris J L 2012 Tailoring fiber grating sensors for assessment of highly refractive fuels Appl. Opt. 51 2015-22

[2] Possetti G R C, Côcco L C, Yamamoto C I, De Arruda L V R, Falate R, Muller M and Fabris J L 2009 Application of a long-period fibre grating-based transducer in the fuel industry Meas. Sci. Technol. 20034012

[3] Vengsarkar A M, Lemaire P J, Judkins J B, Bhatia V, Erdogan T and Sipe J E 1996 Long-period fiber gratings as band-rejection filters J. Lightwave Technol. 14 58-65

[4] Stegall D B and Erdogan T 2000 Dispersion control with use of long-period fiber gratings J. Opt. Soc. Am. A 17 304-12

[5] Beltrán-Mejía F, Cordeiro C M B, Andrés P and Silvestre E 2012 Broadband dispersion compensation using inner cladding modes in photonic crystal fibers Opt. Express $203467-72$

[6] James S W and Tatam R P 2003 Optical fibre long-period grating sensors: characteristics and application Meas. Sci. Technol. 14 R49-61

[7] Chan H M, Alhassen F, Tomov I V and Lee H P 2008 Fabrication and mode identification of compact long-period gratings written by $\mathrm{CO}_{2}$ laser IEEE Photon. Technol. Lett. 20 611-3 
[8] Smietana M, Bock W J and Mikulic P 2010 Comparative study of long-period gratings written in a boron co-doped fiber by an electric arc and UV irradiation Meas. Sci. Technol. 21025309

[9] Savin S, Digonnet M J F, Kino G S and Shaw H J 2000 Tunable mechanically induced long-period fiber grating Opt. Lett. 25 710-2

[10] Lim J, Lee K S, Kim J C and Lee B H 2004 Tunable fiber gratings fabricated in photonic crystal fiber by use of mechanical pressure Opt. Lett. 29 331-3

[11] Cárdenas-Sevilla G A, Monzón-Hernández D, Torres-Gómes I and Martínez-Ríos A 2009 Mechanically induced long-period fiber gratings on tapered fibers Opt. Commun. $2822823-6$

[12] Dianov E M, Vasiliev S A, Kurkov A S, Medvedkov O I and Protopopov V N 1996 In-fiber Mach-Zehnder interferometer based on a pair of long-period gratings ECOC'96: 22nd European Conf. on Optical Communication (Oslo, Norway) vol 1 pp 65-8

[13] Ding J-F, Zhang A P, Shao L-Y, Yan J-H and He S 2005 Fiber-taper seeded long-period grating pair as a highly sensitive refractive-index sensor IEEE Photon. Technol. Lett. 17 1247-9

[14] Cárdenas-Sevilla G A, Monzón-Hernández D, Torres-Gómes I and Martínez-Ríos A 2012 Tapered Mach-Zehnder interferometer based on two mechanically induced long-period gratings as refractive index sensor Opt. Laser Technol. 44 1516-20

[15] Lee B H and Nishii J 1998 Self-interference of long-period fibre grating and its application as temperature sensor Electron. Lett. 34 2059-60
[16] Swart P L 2004 Long-period grating Michelson refractometric sensor Meas. Sci. Technol. 15 1576-80

[17] Barrios P, Sáez-Rodríguez D, Rodríguez A, Cruz J L, Díez A and Andrés M V 2009 Fiber-optic aqueous dipping sensor based on coaxial Michelson modal interferometers J. Sensors 2009 1-4

[18] Kim D W, Zhang Y, Cooper K L and Wang A 2006 Fibre-optic interferometric immuno-sensor using long period grating Eletron. Lett. 42 324-5

[19] Falate R, Kamikawachi R C, Müller M, Kalinowski H J and Fabris J L 2005 Fiber optic sensors for hydrocarbon detection Sensors Actuators B 105 430-6

[20] Heller W 1965 Remarks on refractive index mixtures rules J. Phys. Chem. $691123-9$

[21] Weast R C and Astle M J 1979 Handbook of Chemistry and Physics 59th edn (Boca Raton, FL: CRC Press)

[22] Ran Y, Tan Y-N, Sun L-P, Gao S, Li J, Jin L and Guan B-Ou $2011193 \mathrm{~nm}$ eximer laser inscribed Bragg gratings in microfibers for refractive index sensing Opt. Express 19 18577-83

[23] Kim C-B and Su C B 2004 Measurement of the refractive index of liquids at 1.3 and 1.5 micron using a fibre optic Fresnel ratio meter Meas. Sci. Technol. 15 1683-6

[24] Roy S 1999 Fiber optic sensor for determining adulteration of petrol and diesel by kerosene Sensors Actuators B 55 212-6

[25] Rao Y J and Jackson D A 1996 Recent progress in fibre optic low-coherence interferometry Meas. Sci. Technol. 7 981-99

[26] Kersey A and Berkoff T A 1992 Fiber-optic Bragg-grating differential-temperature sensor Photon. Technol. Lett. $41183-5$ 